http://jmscr.igmpublication.org/home/

ISSN (e)-2347-176x ISSN (p) 2455-0450

crossref DOI: https://dx.doi.org/10.18535/jmscr/v7i8.57

\title{
Effect of Low Level Laser Therapy on Wound Healing After Gingival Depigmentation Procedure by Scalpel Technique
}

\author{
Authors \\ Dr Maya Indurkar, Dr. Ketaki Dhumal \\ GDC Aurangabad
}

\section{Introduction}

Low-level laser treatment, also called 'Soft Laser Therapy' was first introduced by Mester and his colleagues. ${ }^{1}$ These lasers work in the milliwatt range with wavelengths in the red or near infrared spectrum (400-900nm). Low-level lasers do not cut or ablate the tissues. The basic principle of low-level laser therapy (LLLT) is based on the biostimulation or the biomodulation effect which consists of the fact that irradiation at a specific wavelength is able to alter cellular behaviour. ${ }^{2}$ Low level laser is a red light or infrared light whose wave length has a low absorption power in water and is capable of penetrating into soft and hard tissues in a depth of $3 \mathrm{~mm}-15 \mathrm{~mm}$. These lasers act on subcellular photo-receptors, especially the electron transfer chain of the mitochondrial membrane resulting in the enhancement of ATP formation, increase in electrical potential of mitochondrial membrane, activation of the nucleus and its synthesis result into an increase in the driving force to the cells. ${ }^{1}$

Gingival hyper pigmentation is increased pigmentation beyond normally expected degree of the oral mucosa. ${ }^{3}$ The Gingival pigmentation occurs due to non-keratinocytes called as melanocytes. These cells are present in gingival epithelium as in other malpighian epithelia. Melanocytes are dendritic cells located in the basal and spinous layers of the gingival epithelium. They synthesize melanin in organelles called pre-melanosomes or melanosomes. ${ }^{4}$

Gingival depigmentation is a periodontal plastic s urgical procedure whereby the hyper pigmentation is removed or reduced by various techniques. The patient demand for improved esthetics is the first and foremost indication for depigmentation. One of the most

common techniques to beemployed is the surgical removal of pigmentation using scalpels. This procedure involves surgical removal of gingival epithelium with a layer of the underlying connective tissue and allowing the denuded conne ctive tissue to heal by secondary intention. ${ }^{5}$ The raw surface that forms after the surgical procedure may cause discomfort to the patient while speaking or having food. Low level laser therapy can be effectively used in these patients to reduce their postoperative pain and discomfort. The purpose of using LLLT as a part of postoperative therapy is to provide patients with minimal discomfort or pain and a shortened healing period.

\section{Aim}

The aim of the study is to evaluate and compare the effects of LLLT on wound healing after depigmentation procedure using scalpel technique. 
Materials and Methods

8 patients requiring depigmentation procedure were included in the study. It is a split mouth study. The procedure was done under local anesthesia 2\% lignocaine with adrenaline. Depigmentation was performed using number 15 surgical blade.

\section{Inclusion Criteria}

1. Patients between 20 to 45 years of age.

2. Patients with adequate oral hygiene.

3. Patients with oral pigmentation index score of 3 or more given by Dummet and Gupta

4. Patients with a high or very high smile line according to Liebert and Derulle classification for smile line i.e. $2 \mathrm{~mm}$ or more of gingival display during smiling.

\section{Exclusion Criteria}

1. Patients with a habit smoking or tobacco chewing.

2. Pregnant females or lactating mothers.

After completion of the surgical process and bleeding stasis, any of the symmetrical surgical sites was randomly assigned by coin toss method for LLLT using a defocused diode laser at $1 \mathrm{~mm}$ distance for $5 \mathrm{~min}$. The diode laser used had a wavelength of $810 \mathrm{~nm}$ with power setting at $1 \mathrm{~W}$ at continuous mode. To prevent the scattering of light to the opposite side, the control site was covered with periodontal dressing. LLLT procedure was repeated each day until 7th day. Visual analog scale, healing index by landry was scoredon the 3rd, 7th, and 15th day. The surface of gingiva which was not epithelized was assessed using a plaque disclosing agent.

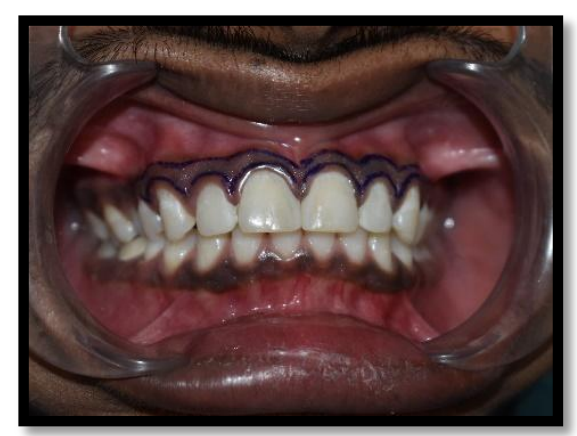

Pre-Operative View

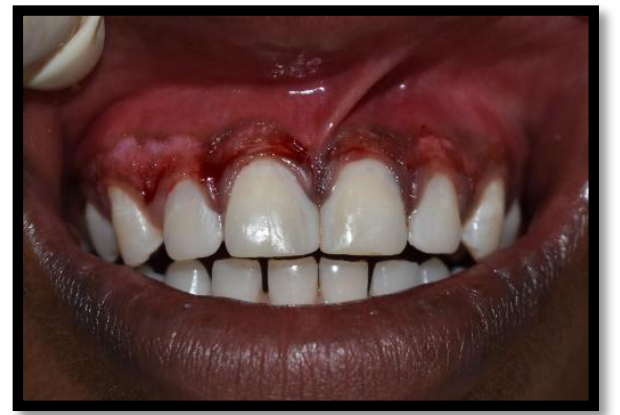

Raw Surface Left Behind After Depigmentation

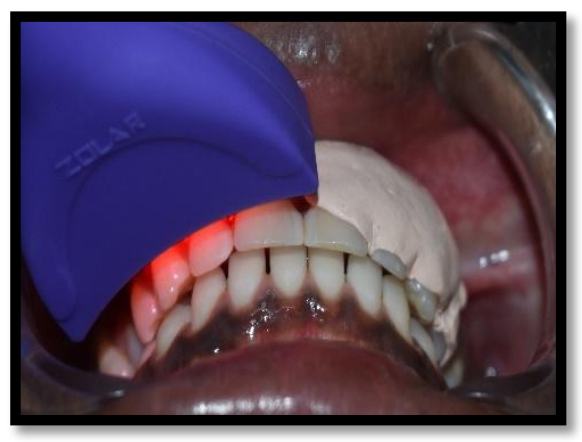

Application of Lllt on the Test Site

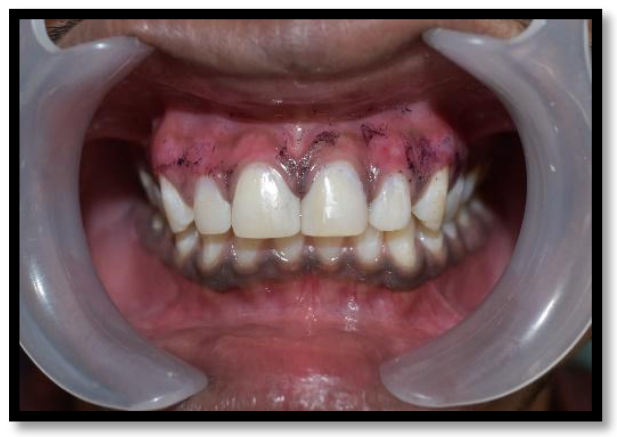

Follow up on $3^{\text {rd }}$ day shows more uptake of disclosing agent on control site

\section{Visual analog scale score determination}

The visual analog scale (VAS) was used to evaluate the subjective pain level experienced by the patient. It consisted of a scale with values ranging from 0 to 10 , with the left end depicting "no pain" and at the right end depicting "unbearable pain." The patients was asked to mark the severity of the pain on this scale. If there was no pain score 0, was recorded. Slight pain was recorded as score, 1 to 3, moderate pain was recorded as score, 3 to 6 , and severe pain was recorded as score, between 6 and 10 . 
Scoring criteria for healing index by landry was as follows

Very poor 1

Tissue color: more than $50 \%$ of gingivae is red; Response to palpation: bleeding; Granulation tissue: present; Incision margin: not epithelialised, with loss of epithelium beyond margins; Suppuration: present

Poor 2

Tissue color: more than $50 \%$ of gingivae is red; Response to palpation: bleeding; Granulation tissue: present; Incision margin: not epithelialised, with connective tissue exposed

\section{Good 3}

Tissue color: less than $50 \%$ of gingivae is red; Response to palpation: no bleeding; Granulation tissue: none; Incision margin: no connective tissue exposed

Very good 4

Tissue color: less than $25 \%$ of gingivae is red; Response to palpation: no bleeding; Granulation tissue: none; Incision margin: no connective tissue exposed

Excellent 5

Tissue color: all gingivae is pink; Response to palpation: no bleeding; Granulation tissue: none; Incision margin: no connective tissue exposed. ${ }^{6}$

Statistical Analysis: For statistical analysis graphpad software was used to obtain mean, standard deviation and $\mathrm{p}$ values.

\section{Results}

\section{Visual Analog Scale Scores}

At day 3, the mean VAS score was $2.62 \pm 0.81$ for the test site and $3.62 \pm 0.45$ for the control site. On the $3^{\text {rd }}$ day, the VAS scores for the test site were less compared to the control site. At day 7, the mean VAS score was $1.62 \pm 0.56$ for the test site and $2.10 \pm 0.78$ for the control site. At day 15 , the patients had no pain on either of the sites. Hence, the VAS scores for both control and test sites were zero. The difference in the VAS scores between the test and the control site was statistically significant; while on the day 7 the VAS scores for test site was less compared to the control site; but the difference was not statistically significant.

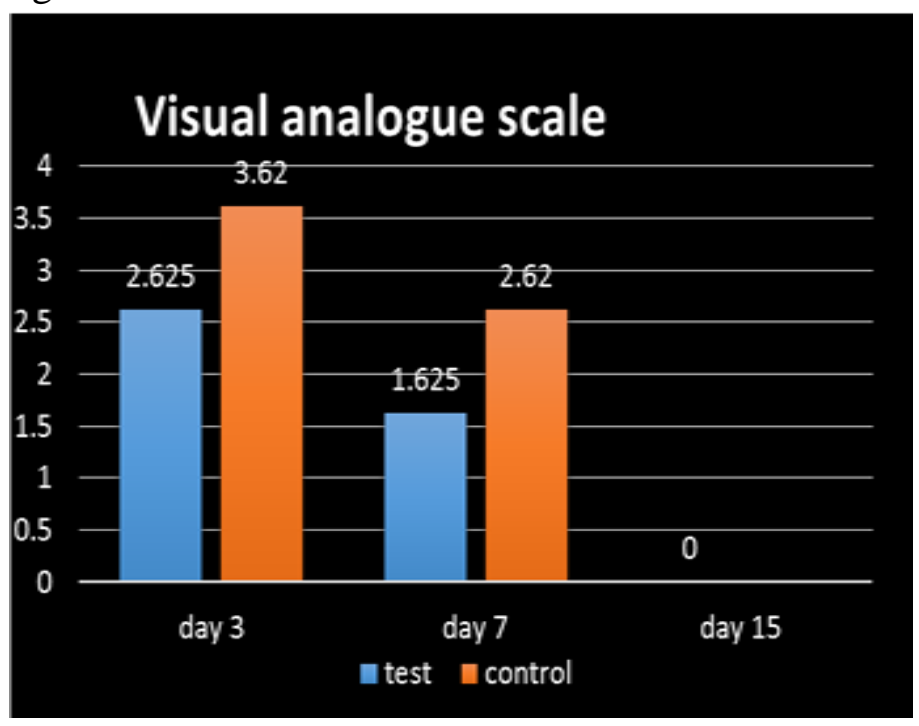

\section{Healing Index by Landry}

At day 3, the mean healing index by landry score was $3.875 \pm 0.81$ for the test site and $2.882 \pm 0.45$ for the control site. At day 7, the mean healing index score was $4.902 \pm 0.56$ for the test site and $3.762 \pm 0.78$ for the control site. At day 15 the scores for the test site and the control site were $5.369 \pm 0.74$ and $5.128 \pm 0.55$ respectively. On the 3rd day, the healing index scores for the test site were less compared to the control site. This difference between the test and the control site was statistically significant; while on the day 7 the healing index scores for test site was less compared to the control site; but the difference was not statistically significant

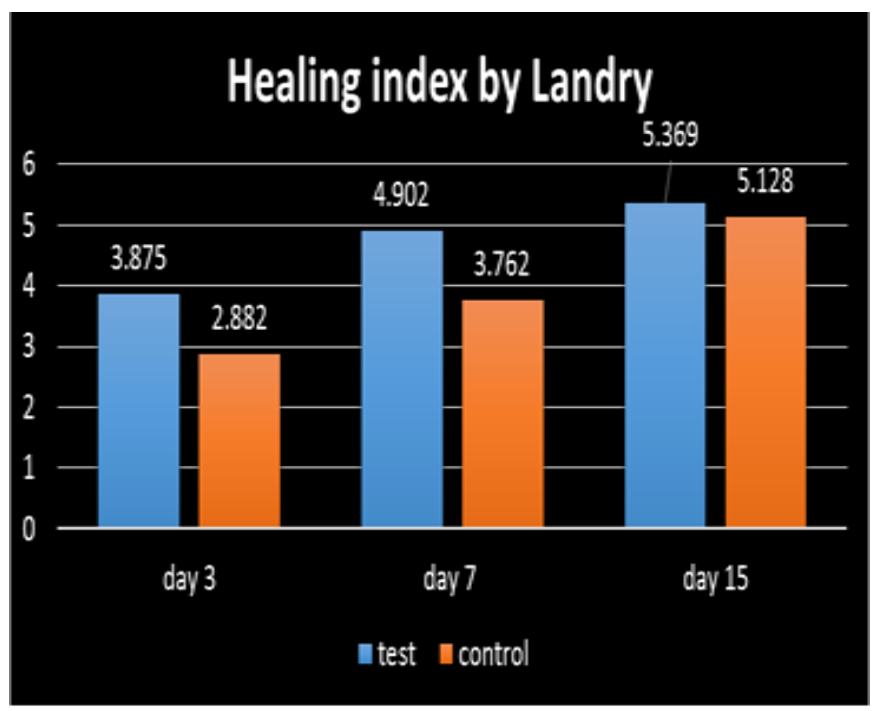




\section{Discussion}

In this clinical trial, the wounds after depigmentation were assessed over 15 days to study whether LLLT improves the healing process and postsurgical patient comfort.

Within the limitations of this study, the findings revealed that LLLT promotes wound healing and also reduces pain perception by the patient after depigmentation procedure until the 3rd day. On the 7th and 15th day, the difference in healing and pain perception was not statistically significant

One possible mechanism by which LLLT may enhance wound healing in vivo is via stimulation of epithelial cells. LLLT increases the motility of human epidermal keratinocytes in vitro, and this would explain the finding that wound sites treated with LLLT show accelerated closure. ${ }^{7}$

The stimulatory effects of LLLT on fibroblast proliferation in vitro are well established. There are several mechanisms by which LLLT may stimulate the proliferation of fibroblasts. LLLT has been shown to stimulate the production of basic fibroblast growth factor (bFGF), a multifunctional polypeptide which supports fibroblast proliferation and differentiation. Fibroblasts irradiated with low dose LLLT show both increased cell proliferation and enhanced production of bFGF, while high dose LLLT suppresses both parameters. A further effect of LLLT on fibroblasts which can influence the wound healing process is the transformation of fibroblasts into myofibroblasts, which are responsible for wound contraction. LLLT of gingival fibroblasts in culture has been shown to induce transformation into myofibroblasts as early as 24 hours after laser treatment. ${ }^{7}$

In vitro and in vivo data suggest that LLLT facilitates fibroblast and keratinocyte cell motility, collagen synthesis, angiogenesis and growth factor release, which lead to increased wound healing. ${ }^{2}$

The ability of LLLT to exert analgesic effects has historically been a major clinical application of the technique. In vivo studies of the analgesic effect of LLLT on nerves supplying the oral cavity have demonstrated that LLLT decreases the firing frequency of nociceptors. ${ }^{7}$

A similar study by ozelick et al which assessed the effects of LLLT on healing of gingiva after gingivectomy and gingivoplasty showed that LLLT may enhance epithelization and improve wound healing after gingivectomy and gingivoplasty operations. These results are comparable to our study.

Another study by kiran Chawla et al .revealed that LLLT promotes wound healing after depigmentation procedure. In this study they had used surface area determination as a parameter instead of healing index as it was used in our study.

\section{Conclusion}

Within the limitations of this study, the findings revealed that LLLT promotes wound healing and decreases pain perception by the patient after depigmentation procedure until the 3rd day. On the 7 th and 15th day, the difference in healing and pain perception was not statistically significant.

\section{References}

1. Sobouti F, Khatami M, Heydari M, Barati M. The Role of Low-Level Laser in Periodontal Surgeries. J Lasers Med Sci 2015;6(2):45-50

2. Ozcelik O, Haytac MC, Kunin A, Seydaoglu G. Improved wound healing by low-level laser irradiation after gingivectomy operations: a controlled clinical pilot study. J Clin Periodontol 2008; 35: 250-254.

3. Chawla K, Lamba AK, Tandon S, Faraz F, Gaba V. Effect of low- level laser therapy on wound healing after depigmentation procedure: A clinical study. J Indian Soc Periodontol 2016;20:184- 8.

4. Carranza Kaustubh P Patil, Vaibhav Joshi, Vijay Waghmode, and Vinayak Kanakdae Gingival depigmentation: A split mouth co mparative study between scalpel and cryo- 
surgery Contemp Clin Dent. 2015 Mar; 6

(Suppl 1): S97-S101

5. Landry R, Turnbull R, Howley $\mathrm{T}$. Effectiveness of benzydamine $\mathrm{HCl}$ in the treatment of periodontal post-surgical patients. Res Clin Forums 1988;10:105118.

6. L. J. Walsh. The current status of low level laser therapy in dentistry. Part 1. Soft tissue applications. Australian Dental Journal 1997;42:(4):247-54. 